\title{
Implications of Qudit Superselection rules for the Theory of Decoherence-free Subsystems
}

\author{
Mark S. Byrd \\ Physics Department, Southern Illinois University, Carbondale, Illinois 62901-4401
}

\begin{abstract}
The use of $d$-state systems, or qudits, in quantum information processing is discussed. Threestate and higher dimensional quantum systems are known to have very different properties from two-state systems, i.e., qubits. In particular there exist qudit states which are not equivalent under local unitary transformations unless a selection rule is violated. This observation is shown to be an important factor in the theory of decoherence-free, or noiseless, subsystems. Experimentally observable consequences and methods for distinguishing these states are also provided, including the explicit construction of new decoherence-free or noiseless subsystems from qutrits. Implications for simulating quantum systems with quantum systems are also discussed.

PACS numbers: 03.67.Pp,03.65.Yz,03.67.Lx,11.30.-j
\end{abstract}

\section{INTRODUCTION}

At present it is not known which experiment will lead to the first reliable, prototypical quantum computing device. Quantum systems with two states, called qubits, are taken to be the basic unit for quantum information processing and storage. However, in practice, these two states are often only two of a larger set of states. Therefore, one may wonder if a higher-dimensional system will eventually be used in its entirety for quantum computing. Higher dimensional quantum systems, which contain $d$ orthogonal states (called $d$-state systems hereafter), have many interesting properties which differ from those systems which have $d=2$ and may have advantages for quantum information processing. For example two threestate systems, or qutrits, can be more entangled than two qubits [1, 2, 3]. d-state systems can also share a larger fraction of their entanglement [4].

In addition to the differences in entanglement properties for quantum systems with more than two orthogonal states, there are differences in the selection rules governing the transitions between states. Some of these selection rules are referred to as "superselection" rules. (See [5] and references therein.) In the present article, a superselection rule will be taken to mean that a system's "principal" quantum numbers cannot be changed in a closed system. A principal quantum number is defined here as one that identifies an irreducible representation (irrep) of a group. An example of such a rule is the preservation of the principal quantum number $j$ which applies when $J^{2}$ is a constant of the motion. The differences in selection rules arise, in part, from the fact that for systems with $d \geq 3$, more than one principle quantum number is required.

Selection rules, including superselection rules, play an important role in quantum theory [6, 7, 8]. They often define a set of physically accessible states within a

*Electronic address: mbyrd@physics.siu.edu particular experiment. Superselection rules could have important consequences for some quantum information processing protocols [5, 9, 10, 11, 12. However, in the case of quantum cryptographic protocols, it has been shown that superselection rules do not aid in their security since these rules can, in principle, be violated [5] . Here, the importance of selection rules for quantum information processing in other realms is explored. We will see that selection rules have important implications for the theory of decoherence-free, or noiseless, subsystems (DFS/NS) 13, 14, 15, 16, 17, 18] (for recent reviews see $[19,20]$ ), a topic which was also mentioned in connection with superselection rules in [12].

A DFS/NS can be described by a set of selection rules which are obeyed by a system-bath interaction. To compute on a DFS/NS, one violates the system-bath selection rule by using externally applied controls. Taking advantage of these selection rules and encoding in a DFS/NS has been shown to enable universal quantum computing on a noiseless subspace using a limited set of interactions. For example for certain DFS/NSs, the Heisenberg exchange interaction alone can be universal [17, 21, 22, 23, 24, 25]. This is quite an advantage in those systems which have readily available exchange interactions, but no other gating operations which are able to be easily or quickly performed. DFS/NSs also show promise for error protection and universal computing when combined with other methods. (See 20] for a review.) One way to do this is to use dynamical decoupling to eliminate the noise on DFS/NS encoded qubits 26, 27]. Such "leakage elimination operations" [26, 27] can be used to prevent coupling of the two-state system whether it is a subspace of an $d$-state system or a logical qubit comprised of a subspace of a set of physical qubits.

In the case of leakage elimination and also the elimination of gating errors in a multistate system [28], controls are used to eliminate interactions between a subsystem (usually a physical or encoded qubit) of a multilevel system and other states in the system. The objective in this article is to explore the possibility of using the entire $d$-state system for quantum information process- 
ing. Computing with physical $d$-state was discussed in Refs. 29, 30 and distillation protocols for physical $d$ state systems were discussed in Ref. 31]. Both of these articles deal with quantum computing using $d$-state systems while the present article concerns encoding quantum information into collective DFS/NS using $d$-state systems. This is done by first describing a connection between quantum selection rules and operator algebras with group representation theory, operator algebras being useful for the description of DFS/NSs 16]. Then, as mentioned above, implications of this for DFS/NS theory are examined.

More specifically, this article is organized as follows. Section II contains conventions and labeling which will provide a basis for the group-theoretical treatment in this article including the definition of different types of selection rules. Section III reviews some formal aspects of decoherence-free, or noiseless, subsystems. Conventions for the choice of basis and eigenvalues for three-state systems are provided in Section IV] These results will then be used in Section $\nabla$ for the construction of DFS/NS from systems having more than two orthogonal states. This includes details of the a decoherence-free, or noiseless, qubit which is constructed from three qutrits and is immune to arbitrary collective errors. The properties which are important for the generalization to higherdimensional systems are also important for the simulation of quantum systems with quantum systems. Simulations and future work are discussed in the concluding section, Section VI Two appendices provide some grouptheoretical definitions, properties of young tableau, singlet states and a basis for $3 \times 3$ matrices which are used in the text.

\section{BACKGROUND}

In Appendix $\$$ several definitions are given which are required in much of the rest of the article. These definitions can also be found Cornwell [32] (with slight differences). The comments in Appendix $\mathrm{A}$ are added to provide some extra explanation and context. For our purposes, it is enough to note that there exist two inequivalent, fundamental, irreducible representations of $S U(d)$ for all $d \geq 3$. Definitions of "inequivalent representations" and "fundamental representations" are two of the definitions provided in Appendix A

For $S U(3)$, the two different fundamental irreps for $S U(3)$ will be denoted by $\mathbf{3}$ and $\overline{\mathbf{3}}$. In general, representations will be denoted by bold-faced numbers with the numbers indicating the dimension of the representation. States within these two irreps will be denoted $|i\rangle$ and $|\bar{i}\rangle$ respectively. Tensor products will be written, for example, as $|i i\rangle(=|i\rangle \otimes|i\rangle),|i \bar{i}\rangle(=|i\rangle \otimes|\bar{i}\rangle)$, etc.

\section{A. Labels for Irreps}

For physical systems, a complete set of labels for the states is quite important since a complete set of labels is required in order to distinguish elements of a complete set of mutually orthonormal states. In this section, such labels are discussed generally and then given explicitly for irreducible representations of $S U(2)$. In Sec. IV further discussion of this point is taken up and explicit labels for $S U(3)$ are given.

Let $U$ be an element of a matrix representation of a Lie group, parameterized by a set of parameters $a_{i} ; U=$ $U\left(a_{i}\right)$. The elements of the matrix may then be denoted:

$$
D_{m, m^{\prime}}^{(r)}\left(a_{i}\right)=\left\langle r, m\left|U\left(a_{i}\right)\right| r, m^{\prime}\right\rangle .
$$

In general, for $d \geq 3 r$ will represent more than one number. Similarly, $m$ and $m^{\prime}$ will each represent more than one number. Quantum numbers $r$ represent "principal quantum numbers" and the quantum numbers $m, m^{\prime}$ will be referred to as "secondary quantum numbers."

Let us give the familiar example of angular momentum in quantum mechanics. The principal quantum number is taken to be $j$ which labels the total angular momentum through its relationship with the eigenvalue of the total angular momentum operator, $J^{2}$ :

$$
J^{2}|j, m\rangle=j(j+1)|j, m\rangle .
$$

If Euler angles $\alpha, \beta, \gamma$ are chosen to parameterize the matrix $U$, then the matrix elements are given by

$$
D_{m, m^{\prime}}^{(j)}(\alpha, \beta, \gamma)=\left\langle j, m|U(\alpha, \beta, \gamma)| j, m^{\prime}\right\rangle .
$$

Here $j$ is the principal quantum number. (Here there is only one.) And $m, m^{\prime}$ label states within an irrep. Transitions may occur which change the $z$ component of the angular momentum $m$, but if $J^{2}$ is a constant of the motion, then $j$ will not change.

These labels let us define a superselection rule as being one for which the principal quantum numbers are conserved; i.e., a superselection rule exists-and is not violated-if one cannot transform a state in one irreducible representation to a state in different irreducible representation. If two such representations are accessible and equivalent, we can include another "principal quantum number" to label this degeneracy.

\section{B. Superselection rules}

To make connection with previous work, note that the definition of a superselection rule used in this article is not significantly different from the one used by [5] and [12] which state that a local superselection rule exists if there is a symmetry in the system. In other words, a superselection rule exists if the system has the property 
that it is invariant under a group of transformations, $\mathcal{G}$ viz.,

$$
|\psi\rangle\left\langle\psi\left|=\int_{\mathcal{G}} U\right| \psi\right\rangle\langle\psi| U^{\dagger} d U
$$

where $U \in \mathcal{G}$ and $d U$ is the group-invariant Haar measure. The existence of such a symmetry implies that the group of all transformations on the Hilbert space is reducible. This divides the space into superselection sectors. Here we identify each superselection sector with an irrep of a group. By our definitions, a superselection rule prevents the system from being transformed from a state within one sector to a state within another.

\section{DECOHERENCE-FREE OR NOISELESS SUBSYSTEMS}

In this section, a brief review of DFS/NS is provided using the notation of Refs. 17] and [27]. This is followed by a statement of a theorem which apparently has not been previously applied to DFS/NS theory and which formally relates group theoretical representation theory to algebraic representation theory. These and the example in the next section provide an application of these methods to a DFS/NS which is known. This section is then followed by new results.

\section{A. Definitions DFS/NS}

Consider a system $S$ coupled to a bath $B$ via the Hamiltonian

$$
H=H_{S} \otimes \mathbb{1}_{B}+\mathbb{1}_{S} \otimes H_{B}+H_{I}
$$

where $H_{S}$ acts only on the system Hilbert space $\mathcal{H}_{S}$, $H_{B}$ acts only on the bath Hilbert space $\mathcal{H}_{B}, \mathbb{1}_{S}$ is the identity operator on the system Hilbert space, $\mathbb{1}_{B}$ is the identity operator on the bath Hilbert space, and $H_{I}$ is the interaction Hamiltonian which acts on both the system and bath Hilbert spaces $\mathcal{H}_{S} \otimes \mathcal{H}_{B}$ and couples the two. In general, $H_{I}$ can be written as a sum of operators which act on the system $\left(S_{\alpha}\right)$ and operators which act on the bath $\left(B_{\alpha}\right)$,

$$
H_{I}=\sum_{\alpha} S_{\alpha} \otimes B_{\alpha}
$$

If there is no interaction Hamiltonian-i.e., when $H_{I}=0$ the system and bath evolve separately and unitarily:

$$
U(t)=\exp \left[-i H_{S} t\right] \otimes \exp \left[-i H_{B} t\right]
$$

where $\hbar=1$.

Consider the (associative) algebra, denoted $\mathcal{A}$, and generated by $H_{S}$ and the set of $S_{\alpha}$. This is a $\dagger$-closed algebra (if $A_{i} \in \mathcal{A}$, then $A_{i}^{\dagger} \in \mathcal{A}$ ) which is, by assumption, reducible. This implies that the algebra is isomorphic to a direct sum of $d_{J} \times d_{J}$ complex matrix algebras, each with multiplicity $n_{J}$ :

$$
\mathcal{A} \cong \underset{J \in \mathcal{J}}{\oplus} \mathbb{1}_{n_{J}} \otimes \mathcal{M}\left(d_{J}, \mathbb{C}\right) .
$$

$\mathcal{J}$ is a finite set labeling the irreducible components of $\mathcal{A}$, and $\mathcal{M}\left(d_{J}, \mathbb{C}\right)$ denotes a $d_{J} \times d_{J}$ complex matrix algebra. The commutant $\mathcal{A}^{\prime}$ of $\mathcal{A}$ is defined by

$$
\mathcal{A}^{\prime}=\{X:[X, A]=0, \forall A \in \mathcal{A}\} .
$$

The elements of this set also form a $\nmid$-closed algebra. This algebra is also reducible, with

$$
\mathcal{A}^{\prime} \cong \underset{J \in \mathcal{J}}{\oplus} \mathcal{M}\left(n_{J}, \mathbb{C}\right) \otimes \mathbb{1}_{d_{J}}
$$

An element of $\mathcal{A}$ can be written in block-diagonal form with $J$ denoting subblocks given in Eq. (7). A typical block with given $J$ can be further decomposed as

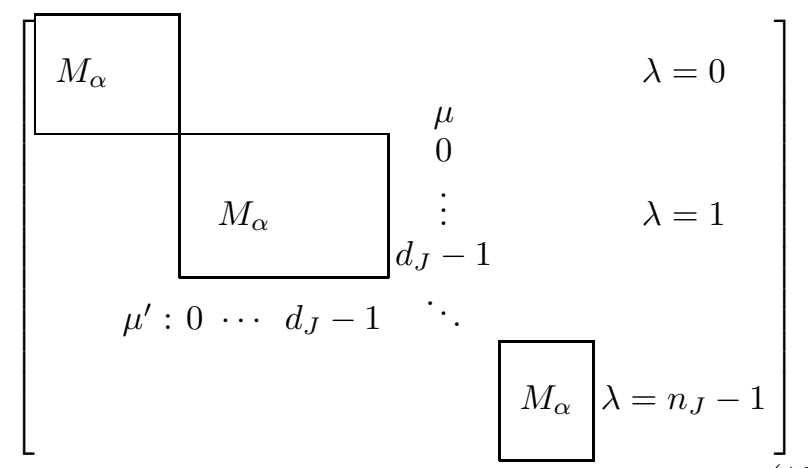

Here $\lambda$ labels the different degenerate subblocks, $0 \leq \lambda \leq$ $n_{J}-1$ and $\mu$ labels the states inside each $\lambda$-subblock, $0 \leq \mu \leq d_{J}-1$. Associated with this decomposition of the algebra $\mathcal{A}$ is the decomposition of the system Hilbert space:

$$
\mathcal{H}_{S}=\sum_{J \in \mathcal{J}} \mathbb{C}^{n_{J}} \otimes \mathbb{C}^{d_{J}} .
$$

Decoherence-free or noiseless subsystems can now be defined. Let $\left\{\left|\lambda_{\mu}\right\rangle\right\}$, denote a subspace of $\mathcal{H}_{S}$ with given $J$. Then the condition for the existence of an irreducible decomposition is

$$
A_{\alpha}\left|\lambda_{\mu}\right\rangle=\sum_{\mu^{\prime}=1}^{d_{J}} M_{\mu \mu^{\prime}, \alpha}\left|\lambda_{\mu^{\prime}}\right\rangle
$$

for all $A_{\alpha}, \lambda$, and $\mu$. Notice that $M_{\mu \mu^{\prime}, \alpha}$ does not depend on $\lambda$. Thus for a fixed $\lambda$, the subspace spanned by $\left|\lambda_{\mu}\right\rangle$ is acted upon in some nontrivial way. However, because $M_{\mu \mu^{\prime}, \alpha}$ is not dependent on $\lambda$, each subspace defined by a fixed $\mu$ and running over $\lambda$ is acted upon in an identical manner by the decoherence process. The information is stored in blocks with the same $J$, but different $\lambda$, and this defines a DFS/NS. Therefore, the labels which define the decoherence-free, or noiseless, states are the $\lambda$. 
A decoherence-free or noiseless subspace is one for which the matrices $M_{\mu \mu^{\prime}, \alpha}$ are one by one, i.e., they are numbers. If the $M$ are numbers $(1 \times 1$ matrices $)$, then they act on a $1 \times 1$ representation, which is necessarily a singlet state (a one-dimensional representation).

\section{B. Weyl's unitary trick}

In the following sections, group theoretical methods will be used to identify DFS/NSs. In particular, the representation theory of $S U(d)$ will be used repeatedly in order to find degeneracies which are able to represent DFS/NSs. It is not immediately obvious that there exists an equivalence between group representation theory and the algebraic representation theory above. In other words, it may not be clear that the representation theory of the algebra of complex matrices is directly related to the theory of representations of the unitary groups. However, the representations are directly related and the use of this relation is sometimes referred to as Weyl's unitary trick. The theorem from Huang [33] is stated here without proof. (For a proof, see 33.)

Weyl's unitary trick. The following sets of representations on finite vector spaces are in one to one correspondence. Moreover, under the correspondence, invariant subspaces and equivalences are preserved.

(i) holomorphic representations of $S L(d, \mathbb{C})$;

(ii) representations of $S L(d, \mathbb{R})$

(iii) representations of $S U(d, \mathbb{R})$

(iv) representations of $\operatorname{sl}(d, \mathbb{R})$

(v) representations of $s u(d)$

(vi) complex linear representations of $\operatorname{sl}(d, \mathbb{C})$

Here a holomorphic (analytic) representation of $S L(d, \mathbb{C})$ is defined to be a homomorphism which is also a holomorphic map. The lowercase letters designate an algebra rather than a group. For example, su(2) is the Lie algebra of the group $S U(2)$.

As an application of this "trick," let us consider collective decoherence effects. These are noises which act identically on every physical state in the system. Let $\pi\left(L^{\beta}\right)$ a representation of a basis element of an abstract algebraic element $L^{\beta}$. Let $\left|a_{i}\right\rangle$ and their tensor products carry a representation of a group $G$ generated by the set $\left\{L^{\beta}\right\}$, denoted $\mathcal{L}$. Then

$$
\begin{aligned}
\pi_{e}\left(L^{\beta}\right)\left(\left|a_{1}\right\rangle \otimes \mid\right. & \left.\left|a_{2}\right\rangle \otimes \cdots\left|a_{m}\right\rangle\right) \\
=[ & \left.\pi_{1}\left(L^{\beta}\right)\left|a_{1}\right\rangle\right] \otimes\left(\left|a_{2}\right\rangle \otimes \cdots\left|a_{m}\right\rangle\right) \\
& +\left|a_{1}\right\rangle \otimes\left[\pi_{2}\left(L^{\beta}\right]\left|a_{2}\right\rangle\right) \otimes \cdots\left|a_{m}\right\rangle \\
& +\cdots \\
& +\left|a_{1}\right\rangle \otimes\left|a_{2}\right\rangle \otimes \cdots\left[\pi_{m}\left(L^{\beta}\right)\left|a_{m}\right\rangle\right],
\end{aligned}
$$

where $\pi_{e}$ is the representation on the entire space and $\pi_{i}$ is the representation on the $i^{t h}$ subsystem. The algebra acting this way corresponds to the quantum numbers being additive. For collective decoherence on a set of $m$ physical qudits, each $\pi_{i}\left(L^{\beta}\right)$ is identical. This provides a correspondence between the algebraic elements $L^{\beta}$ acting on the group and the algebraic elements which act as noises on the states and establishes the relation between tensor products of representations and direct sums of representations.

To exemplify and clarify these statements, the threequbit DFS/NS is reexamined in the following section. This will show how to provide generalizations of these operators, and the corresponding DFS/NSs to $d$-state systems.

\section{Example: Three-qubit DFS/NS}

The review of the three-qubit noiseless subsystem will enable the introduction of some general techniques, including Young tableau, in a more familiar context. (Rules for using Young tableau are given in [34, 35] and briefly discussed in Appendix A] In this case a decoherencefree, or noiseless, subsystem is formed from two doublet states in the Hilbert space of three two-state systems. This code protects a single two-state subspace, referred to as the encoded qubit, from collective errors.

Let us use Young's tableau to find the doublets. For qubits, $d=2$, so there are two possible numbers with which boxes of a Young diagram can be filled. Let us consider the following example of one box:

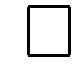

Filling this with either a 1 or a 2, implies

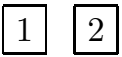

This is a doublet, or two-dimensional representation of $S U(2)$. Taking the product gives

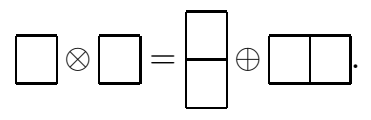

These can be filled in the following ways according to the rules for using Young's tableau. The first can only have

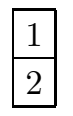

The second can have

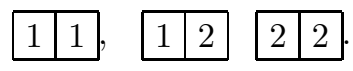

which gives a singlet and a triplet respectively. This can be summarized in the equation $\mathbf{2} \otimes \mathbf{2}=\mathbf{3} \oplus \mathbf{1}$. Taking the 
tensor products of three doublets,

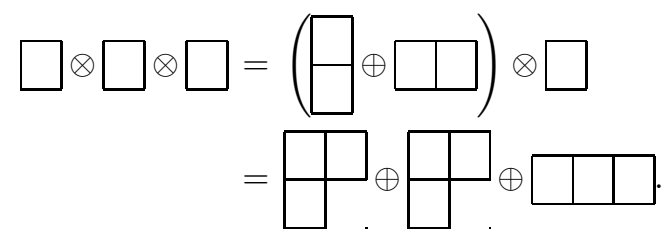

Filling in the numbers implies that there are two doublets and a quadruplet state in the direct sum decomposition, giving a total of eight states. (Note that the set of three vertical boxes which one might have drawn here is not present. This is because there is no nonzero state with three antisymmetric indices.)

Now, the following convention is used for the computational basis states $|0\rangle$ and $|1\rangle:|0\rangle=|1 / 2,1 / 2\rangle,|1\rangle=$ $|1 / 2,-1 / 2\rangle$. These are the two states of a single spin- $1 / 2$ particle, or a representation of the $j=1 / 2$ representation of $S U(2)$. This convention is opposite to that of Ref. [17], but follows the conventions of [27], both of which provide more detail than is given here. Three-qubit DFS/NS encoded qubit will now be represented in the following way,

$$
\left(\begin{array}{c}
(|010\rangle-|100\rangle) / \sqrt{2} \\
(|011\rangle-|101\rangle) / \sqrt{2} \\
(2|001\rangle-|010\rangle-|100\rangle) / \sqrt{6} \\
(-2|110\rangle+|011\rangle+|101\rangle) / \sqrt{6} \\
|000\rangle \\
(|001\rangle+|010\rangle+|100\rangle) / \sqrt{3} \\
(|011\rangle+|101\rangle+|110\rangle) / \sqrt{3} \\
|111\rangle
\end{array}\right\} \mathcal{C}^{\perp}
$$

With this notation $\left|0_{L}\right\rangle=\alpha_{0}(|010\rangle-|100\rangle) / \sqrt{2}+$ $\beta_{0}(|011\rangle-|101\rangle) / \sqrt{2}$ (arbitrary superposition), and likewise $\left|1_{L}\right\rangle=\alpha_{1}(2|001\rangle-|010\rangle-|100\rangle) / \sqrt{6}+\beta_{1}(-2|110\rangle+$ $|011\rangle+|101\rangle) / \sqrt{6}$. These states belong to the two $J=1 / 2$ irreps of $S U(2)$. The coefficients are WignerClebsch-Gordan coefficients [36] and the last four states comprise a $J=3 / 2$ representation of $S U(2)$. The two $J=1 / 2$ irreps can be distinguished by a degeneracy label $\lambda=0,1$. Thus a basis state in the eightdimensional Hilbert space is fully identified by the three quantum numbers $|J, \lambda, \mu\rangle$, where $\mu$ is the $z$-component of the total spin $J$. In this notation we can write $\left|0_{L}\right\rangle=\alpha_{0}|1 / 2,0,1 / 2\rangle+\beta_{0}|1 / 2,0,-1 / 2\rangle$ and $\left|1_{L}\right\rangle=$ $\alpha_{1}|1 / 2,1,1 / 2\rangle+\beta_{1}|1 / 2,1,-1 / 2\rangle$.

If this encoded qubit is affected by collective errors-i.e., errors that act the same on each physical qubit-then no information is lost to the environment. The collective errors are formed from linear combinations of the operators $S^{\alpha}=\sum_{i} \sigma_{i}^{\alpha}$ :

$$
S=\sum_{\alpha} a_{\alpha} S^{\alpha}
$$

where $\alpha=x, y$, or $z$ and the $i$ denotes the physical qubit 1,2 , or 3 . The states within blocks $\left|0_{L}\right\rangle$ and $\left|1_{L}\right\rangle$ couple in exactly the same way, but neither block couples with states outside of that block. Logical operations create superpositions of these blocks. This can be described in terms of group representation theory, using Weyl's unitary trick. A basis for the algebra which spans the space of collective errors can be chosen to be the Lie algebra of $S U(2), s u(2)$. If we consider the action of the algebra on the entire space of the three qubits and suppose that this is a representation of $s u(2)$ as well, then the representation on the entire space of three qubits is affected by the same operation on each physical qubit. This is the statement made generally in Eq. (13).

For the example of three qubits, the matrix, Eq. (10), can be found using the DFS/NS transformation [Eq. (18) of [27]]. In the DFS/NS basis the explicit form is given by

$$
\begin{aligned}
S_{\mathrm{dfs}} & =U_{\mathrm{dfs}} S U_{\mathrm{dfs}}^{-1} \\
& =\left(\begin{array}{cccccccc}
a_{3} & a_{1}-i a_{2} & 0 & 0 & 0 & 0 & 0 & 0 \\
a_{1}+i a_{2} & -a_{3} & 0 & 0 & 0 & 0 & 0 & 0 \\
0 & 0 & a_{3} & a_{1}-i a_{2} & 0 & 0 & 0 & 0 \\
0 & 0 & a_{1}+i a_{2} & -a_{3} & 0 & 0 & 0 & 0 \\
0 & 0 & 0 & 0 & 3 a_{3} & \sqrt{3}\left(a_{1}-i a_{2}\right) & 0 & 0 \\
0 & 0 & 0 & 0 & \sqrt{3}\left(a_{1}+i a_{2}\right) & a_{3} & 2\left(a_{1}-i a_{2}\right) & 0 \\
0 & 0 & 0 & 0 & 0 & 2\left(a_{1}+i a_{2}\right) & a_{3} & \sqrt{3}\left(a_{1}-i a_{2}\right) \\
0 & 0 & 0 & 0 & 0 & 0 & \sqrt{3}\left(a_{1}+i a_{2}\right) & -3 a_{3}
\end{array}\right) .
\end{aligned}
$$

Thus we see that the two states of the logical zero transform in exactly the same way as the two states of the logical one under collective operations.

In the context of this example, let us also consider 
a collection of physical qubits. When collective errors occur, the form of these errors is

$$
\pi_{e}\left(L^{\beta}\right)=\sum_{i} \pi_{i}\left(L^{\beta}\right)
$$

where $L^{\beta}$ is an element of the algebra and the subscript identifies the error as acting on the $i^{t h}$ physical qubit. In other words, the operator $L^{\beta}$ acts on the system of qubits by acting with $L_{1}^{\beta}$ on qubit $1, L_{2}^{\beta}$ on qubit 2 , etc. Each $L_{i}^{\beta}$ is identical, but acts on a different two-state system. Therefore, the statement that collective errors occur, is the statement that the entire system of qubits transforms as a representation of $s u(2)$. By the unitary trick, there is a direct correspondence between the representation theory of the group $S U(2)$ and the representation theory of the algebra with complex coefficients. In this case, the $S U(2)$ transformation of the direct product of three two-dimensional representations is expressed as the direct sum of two three-dimensional representations (and a four-dimensional representation).

This perspective of collective errors will now be used in the following sections to describe DFS/NSs of higher dimensional Hilbert spaces. Though the arguments here are primarily restricted to the concrete example of $S U(3)$, they can readily be extended to any $S U(d)$.

\section{TYPES OF QUTRIT STATES AND LABELING}

In this section explicit labels are provided for the states of qutrits. As noted in Appendix $\mathrm{A}$ and also Sec. II two different types of qutrit states exist. This is true independent of the basis chosen for the algebra. Usually different sets of bases are chosen which reflect the symmetry of the physical system. For example, given a representation of $S U(3)$, there are different subgroup chains which provide different possibilities for sets of measurements, corresponding to a different choice of basis elements,

$$
\begin{gathered}
S U(3) \supset S U(2) \supset U(1) \\
S U(3) \supset S O(3) \supset S O(2) .
\end{gathered}
$$

The first is used in particle physics to describe the three lightest flavors of quarks 37. Associated with this subgroup chain is the set of Gell-Mann matrices. The second of these subgroup chains is used in nuclear physics models, such as that of Elliott [38]. The appropriate set of measurements depends on the "good" quantum numbers of the system.

To each of these subgroup chains there corresponds a complete set of commuting operators (CSCO). These operators are simultaneous observables which can be used to distinguish the different states within an irrep. In the remainder of this article, subgroup chain (19) is considered almost exclusively although the arguments can be applied to chain (20) as well. By using this example, we are able to discuss the importance of the existence of two inequivalent fundamental irreps of the $S U(d)$ groups, both in the theory of DFS/NS and also in simulating quantum systems with quantum systems.

\section{Labels}

As stated above, each of the two types irreps will be associated with the first subgroup chain (19). These will be called qutrit and barred states. Qutrit states will be associated with the $\mathbf{3}$ representation and barred states will be associated with $\overline{\mathbf{3}}$, which is the complex conjugate of the $\mathbf{3}$ rep. Throughout the rest of the article, states in the $\overline{\mathbf{3}}$ rep will have a bar over them to distinguish them from states in the $\mathbf{3}$ rep: for example, the states $|0\rangle,|1\rangle,|2\rangle \in \mathbf{3}$ and $|\overline{0}\rangle,|\overline{1}\rangle,|\overline{2}\rangle \in \overline{\mathbf{3}}$.

In order to provide a complete set of labels which distinguish two orthonormal states, a CSCO must be measured [36]. For irreps of $S U(3)$, the following set of labels completely describe states within an irrep. Each is associated with an operator in the CSCO. Let $p, q$ label the irrep and $t$ label the eigenvalue of an $S U(2)$ subgroup of $S U(3) ; T^{2}|\psi\rangle=t(t+1)|\psi\rangle$ for $|\psi\rangle$ an eigenstate of the operator $T^{2}$. (Lowercase letters will represent the eigenvalues of the operators which will be denoted with an uppercase.) The symbol $t_{3}$ will denote the eigenvalue of $T_{3}$, and $y$ will denote the eigenvalue of the operator $Y$. In terms of the Gell-Mann matrices, (see Appendix B)

$$
\begin{gathered}
Y=\frac{1}{\sqrt{3}} \lambda_{8}, \\
T^{2}=\frac{1}{4}\left(\lambda_{1}^{2}+\lambda_{2}^{2}+\lambda_{3}^{2}\right), \\
T_{3}=\frac{1}{2} \lambda_{3} .
\end{gathered}
$$

The quantum numbers $p$ and $q$ can be determined by the highest-weight states (described later in this section) or by measurement of the Casimir operators. (See Appendix (B) The Casimir operators, plus the set of operators in Eq.(21) provide a CSCO. States within any irreps can be written as $\left|p, q, t, t_{3}, y\right\rangle$, where $p$ and $q$ are assumed to be fixed and determined by the irrep. To make a connection with the more familiar case of a spin- $j$ particle, $p, q$ should be considered "principal" quantum numbers which label an irrep. When $J^{2}$ is a constant of the motion, then $j$ is fixed and cannot change. The analog for three-state systems is the conservation of the two quantum numbers $p$ and $q$. It will be assumed here, unless otherwise stated, that $p$ and $q$ are both conserved. However, whether or not these quantum numbers are conserved in a particular experiment depends on the physical system in question.

The comparison with the unitary representation of the group is made by labeling the unitary matrices in the following way:

$$
\left\langle p, q ; t, t_{3}, y|U| p, q ; t^{\prime}, t_{3}^{\prime}, y^{\prime}\right\rangle=D_{t, t_{3}, y ; t^{\prime}, t_{3}^{\prime}, y^{\prime}}^{(p, q)}
$$



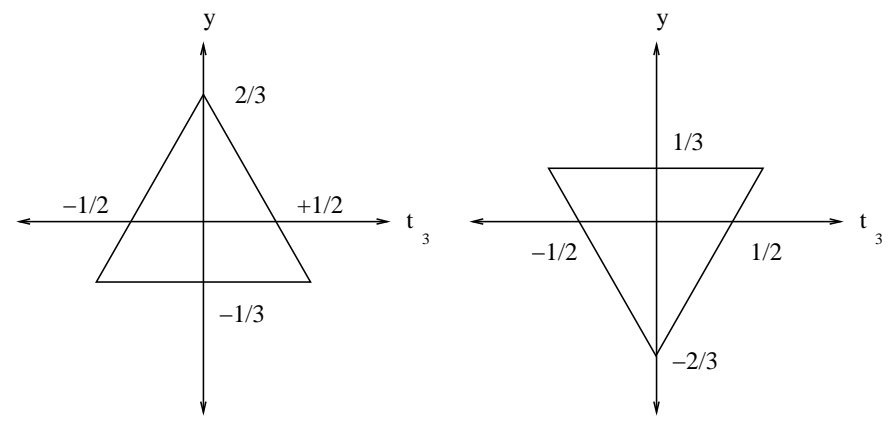

FIG. 1: State spaces for the $\mathbf{3}$ (left) and $\overline{\mathbf{3}}$ (right) reps.

so that the matrix elements of $U$ are given by the functions $D$ and the rows (columns) are labeled by the primed (unprimed) numbers.

For $S U(3)$ there are six raising and lowering operators which take one state in an irrep to another state in the same irrep. They are often denoted $U_{ \pm}, V_{ \pm}, T_{ \pm}$.

Within an irrep, one can define a unique "maximum weight state" (see [35, 39]). This state $\left|\psi_{m}\right\rangle$ is usually defined as the one for which the following relations hold:

$$
T_{+}\left|\psi_{m}\right\rangle=0, \quad V_{+}\left|\psi_{m}\right\rangle=0, \quad U_{-}\left|\psi_{m}\right\rangle=0 .
$$

Since the maximum weight state is unique for each irrep, it can be related to the labels, $p$ and $q$, which identify the irrep,

$$
t_{3 m}=\frac{p+q}{2}, \quad y_{m}=\frac{p-q}{3} .
$$

The examples of the two fundamental irreps $\mathbf{3}$ and $\overline{\mathbf{3}}$ are given in Fig. 1] where the states are labeled according to the eigenvalues of $Y$ and $T_{3}$. The highest weight states in $\mathbf{3}$ and $\overline{\mathbf{3}}$ are the states with $t_{3}=1 / 2, y=-1 / 3$ and $t_{3}=1 / 2, y=1 / 3$ respectively.

Let us now label the states $|0\rangle,|1\rangle,|2\rangle$ by using the full set of quantum numbers,

$$
\begin{aligned}
& |0\rangle=|1,0,1 / 2,1 / 2,1 / 3\rangle, \\
& |1\rangle=|1,0,1 / 2,-1 / 2,1 / 3\rangle, \\
& |2\rangle=|1,0,0,0,-2 / 3\rangle .
\end{aligned}
$$

and

$$
\begin{aligned}
|\overline{0}\rangle & =|0,1,-1 / 2,-1 / 2,-1 / 3\rangle, \\
|\overline{1}\rangle & =|0,1,-1 / 2,1 / 2,-1 / 3\rangle, \\
|\overline{2}\rangle & =|0,1,0,0,2 / 3\rangle .
\end{aligned}
$$

For future reference, note that an $S U(3)$ singlet state has the unique property that

$$
T_{ \pm}\left|\psi_{s}\right\rangle=0, \quad V_{ \pm}\left|\psi_{s}\right\rangle=0, \quad U_{ \pm}\left|\psi_{s}\right\rangle=0 .
$$

This follows from the fact that a "singlet" state is one for which there exists only one state within the irrep (see also Appendix A3 and the comment at the end of Sec. III A).

\section{DECOHERENCE-FREE SUBSPACES FOR THREE-STATE SYSTEMS}

In principle, one can find DFS/NSs from the formal theory provided in [16]. However, until now, there has been no emphasis on the implications of the various irreps of a given group (with the exception of [12]). Here, in particular, it is shown that the distinction between the $\mathbf{3}$ and $\overline{\mathbf{3}}$ representations is tantamount to the identification and use of a DFS/NS for quantum error avoidance in qutrit systems.

Again, in what follows three-state systems are used as explicit examples. However, the constructions here are readily generalizable to $S U(d)$.

\section{A. Product states}

It is common in the quantum information literature to find statements such as "... the maximally entangled state of two qutrits, $|\phi\rangle=\frac{1}{\sqrt{3}}(|00\rangle+|11\rangle+|22\rangle)$." However, we have just seen that there are two different irreducible fundamental representations of $S U(3)$. In that case, we should distinguish between this state, $|\phi\rangle$ and $\left|\phi^{\prime}\right\rangle=\frac{1}{\sqrt{3}}(|0 \overline{0}\rangle+|1 \overline{1}\rangle+|2 \overline{2}\rangle)$. This observation has an important consequence since there is a striking difference between these two states. The state $\left|\phi^{\prime}\right\rangle$ is an $S U(3)$ singlet, but $|\phi\rangle$ is not. Singlet states are used in the theory of DFS in order to protect against all forms of errors. The state $|\phi\rangle$ is not decoherence free. (To see that $\left|\phi^{\prime}\right\rangle$ is a singlet state, consult Appendix A3 )

Note that, in principle, a local unitary can be found which will transform the state $|\phi\rangle$ into the state $\left|\phi^{\prime}\right\rangle$. This implies that the amount of entanglement in $|\phi\rangle$ is the same as the amount of entanglement in $\left|\phi^{\prime}\right\rangle$. Therefore, whereas it has been conjectured that better quantum error correcting codes are those with more entanglement present in their states [40], no such correspondence can be made for DFS/NS. In other words, there is no direct correspondence between the amount of entanglement in a DFS/NS and the efficacy, or error avoidance properties, of the encoded DFS/NS states. This is indicated from the example just presented as well as the corresponding Young diagram wich applies to any two $d$-state systems.

The states $|\phi\rangle$ and $\left|\phi^{\prime}\right\rangle$, belong to two different irreps. Checking all quantum numbers shows that each of the two states has all secondary quantum numbers, $t, t_{3}$, and $y$ equal to zero; that is, $t=0, t_{3}=0$, and $y=0$. However, the primary quantum numbers differ. $|\phi\rangle$ has $p=1, q=0$ whereas $\left|\phi^{\prime}\right\rangle, p=0, q=1$. This difference is experimentally measurable in several different ways. One way is to find the highest weight state of the irrep through the use of the appropriate raising and/or lowering operators. This will identify $p$ and $q$ through Eqs. (23). Also, the differentiation between these states has implications for quantum error avoidance properties of the states. This provides another important method for experimental distinction. 


\section{B. Young Tableau and DFSs}

The use of Young's tableau proves very convenient for exploring the possibility of constructing collective DFS/NSs from qutrits (or qudits). When two or more irreducible representations occur in a tensor product of a set of states of any dimensions, these identical irreps will transform in the same way under an $S U(3)$ action on the entire space of physical subsystems and are therefore candidates for a DFS/NS. (See Section III). Let us now examine some tensor products of qutrits to determine the possibility of constructing DFS/NSs which are immune to collective errors.

The two different Young tableau for the $\mathbf{3}$ and $\overline{\mathbf{3}}$ representations are represented by

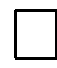

which is filled with numbers $1,2,3$

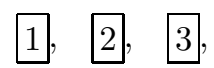

and

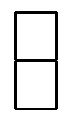

which is filled with numbers $1,2,3$ but is antisymmetric in the interchange of two rows. This gives the following possibilities:

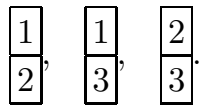

These are the two inequivalent fundamental irreps.

The tensor product of two $\mathbf{3}$ gives the following

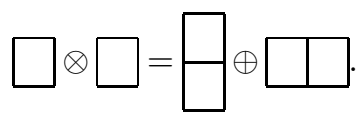

The first is the $\overline{\mathbf{3}}$ rep and the second is a six-dimensional representation which can be shown by filling in the boxes with all possible symmetric combinations

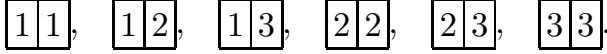

To be precise, this is the 6 rep. The result of this can be written in the following equation: $\mathbf{3} \otimes \mathbf{3}=\overline{\mathbf{3}} \oplus \mathbf{6}$.

Now, note that the result of the product of $\overline{\mathbf{3}}$ and $\mathbf{3}$ is given by

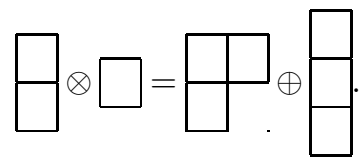

The first tableau corresponds to an octet of states,

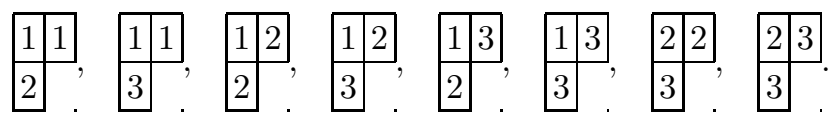

The second corresponds to a singlet, as it can only be filled in one way,

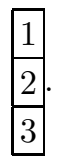

Therefore, $\overline{\mathbf{3}} \otimes \mathbf{3}=\mathbf{8} \oplus \mathbf{1}[41]$. This shows that the product of two three-dimensional representations of the same type do not give rise to a singlet state, whereas products of two reps of different types do give rise to a singlet state. Singlet states are decoherence-free since they are annihilated by all $S U(3)$ operators [15].

Let us consider constructing a decoherence-free, or noiseless, qubit from qutrits. We have already seen this is not possible using two identical qutrits, or an unbarred and a barred rep. One may naturally ask about three unbarred (or three barred) states. From the tableau, it can be shown that $\mathbf{3} \otimes \mathbf{3} \otimes \mathbf{3}=\mathbf{8} \oplus \mathbf{8} \oplus \mathbf{1} \oplus \mathbf{1 0}$. This indicates that three qutrit states have a set of two degenerate reps. This implies that a DFS/NS can be constructed with the two degenerate states representing the logical zero and logical one states of a qubit which is immune to collective noise.

Note, however, that the product of a barred and two unbarred reps will have the following decomposition: $\overline{\mathbf{3}} \otimes$ $\mathbf{3} \otimes \mathbf{3}=\mathbf{1 5} \oplus \mathbf{3} \oplus \mathbf{3} \oplus \overline{\mathbf{6}}$. This indicates that one may also construct a DFS/NS from this set of states which can represent a decoherence-free qubit. Certainly these two are quite different subsystems. The first has two degenerate eight-state subsystems and the second has two degenerate three-state systems.

In order to find the fewest number of physical qutrits which can be encoded such that a logical qutrit is protected from collective errors, four qutrits are taken: $\mathbf{3} \otimes \mathbf{3} \otimes \mathbf{3} \otimes \mathbf{3}=\mathbf{3} \oplus \mathbf{3} \oplus \mathbf{3} \oplus \overline{\mathbf{6}} \oplus \overline{\mathbf{6}} \oplus \mathbf{1 5} \oplus \mathbf{1 5} \oplus \mathbf{1 5} \oplus \overline{\mathbf{1 5}}$. In this case, a decoherence-free qutrit could be represented by three three-state systems and this is the smallest number of qutrit states which can represent such a qutrit DFS/NS.

The analysis can be used for any $d$-state systems. For example, one may ask for the least number of physical $d$-state systems which can be used to encode a logical qubit which is decoherence free with respect to collective errors. The answer can be found by again using Young tableau. The tensor product of three d-state systems can be used to encode logical qubit into a NS/DFS. This can be seen in the tableau of any $\mathbf{d}$ representation

Taking the tensor product of three such systems, pro- 
duces the following tableau

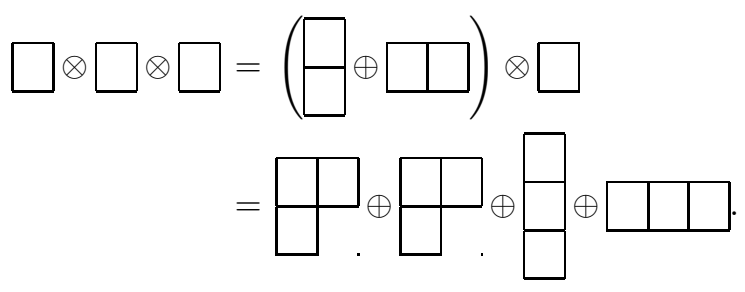

Therefore, this is the smallest number of qudits for which a collective DFS/NS, representing a qubit in terms of qudits, exists.

The difference between a tensor product of two fundamental irreps which are equivalent and two which are not is clearly very important for constructing DFS/NS from higher dimensional systems. The fact that one of the two states transforms differently than the other implies that a superselection rule which preserves the type of qutrit (or qudit) must exist in the system-bath interaction. On the other hand, if one wants to create a DFS/NS by the use of decoupling controls according to the methods presented in 25, 26, 42, 43, 44, 45, 46, 47, 48, 49, 50, then one must recognize this as a quantum control problem in which the decoupling controls must provide the appropriate symmetry for those systems which do not otherwise obey the required superselection rule. In other words, to create a DFS/NS from two inequivalent fundamental irreps, one must ensure that the appropriate transformation properties are obeyed. Representing decoherence-free systems with $d$-state systems therefore requires knowledge of the transformation properties induced by experimental controls and system-bath interactions.

\section{Three-qutrit DFS/NS}

As discussed in the previous section, Sec. $\mathrm{VB}$ a noiseless subsystem can be formed from two octets in the Hilbert space of three three-state systems. This logical qubit will be protected against arbitrary collective errors [see Eq. (27)].

Using the conventions established by Eqs.(24), the logical states can be given explicit labels according to the principal quantum numbers $p, q$ and eigenvalues of the operators $T, Y$, and $T_{3}$. The first of two octets will have a degeneracy label 0 , which indicates that it forms the logical zero state $\left|0_{L}\right\rangle$,

$$
\begin{aligned}
\psi_{1}^{8,0}= & (|200\rangle-|020\rangle) / \sqrt{2}, \\
\psi_{2}^{8,0}= & (|100\rangle-|010\rangle) / \sqrt{2}, \\
\psi_{3}^{8,0}= & (|011\rangle-|101\rangle) / \sqrt{2}, \\
\psi_{4}^{8,0}= & (|211\rangle-|121\rangle) / \sqrt{2}, \\
\psi_{5}^{8,0}= & (|212\rangle-|122\rangle) / \sqrt{2}, \\
\psi_{6}^{8,0}= & (|022\rangle-|202\rangle) / \sqrt{2}, \\
\psi_{7}^{8,0}= & (-|021\rangle-|120\rangle+|201\rangle+|210\rangle) / 2, \\
\psi_{8}^{8,0}= & (2|012\rangle+|021\rangle-2|102\rangle \\
& -|120\rangle-|201\rangle+|210\rangle) / \sqrt{12} .
\end{aligned}
$$

The second octet of states carries a degeneracy label 1 and forms the logical one state $\left|1_{L}\right\rangle$,

$$
\begin{aligned}
\psi_{1}^{8,1}= & (2|002\rangle-|020\rangle-|200\rangle) / \sqrt{6} \\
\psi_{2}^{8,1}= & (2|001\rangle-|010\rangle-|100\rangle) / \sqrt{6} \\
\psi_{3}^{8,1}= & (-2|110\rangle+|011\rangle+|101\rangle) / \sqrt{6} \\
\psi_{4}^{8,1}= & (2|112\rangle-|121\rangle-|211\rangle) / \sqrt{6} \\
\psi_{5}^{8,1}= & (-2|221\rangle+|122\rangle+|212\rangle) / \sqrt{6} \\
\psi_{6}^{8,1}= & (-2|220\rangle+|022\rangle+|202\rangle) / \sqrt{6} \\
\psi_{7}^{8,1}= & (2|012\rangle-|021\rangle+2|102\rangle \\
& \quad-|120\rangle-|201\rangle-|210\rangle) / \sqrt{12} \\
\psi_{8}^{8,1}= & (-|021\rangle+|120\rangle-|201\rangle+|210\rangle) / 2 .
\end{aligned}
$$

In other words, the first superscript denotes the dimension of the representation, the second is a degeneracy label and the subscript labels the state within the representation.

As in the case of the three-qubit DFS/NS, the logical zero state is given by $\left|0_{L}\right\rangle=\sum_{i} \alpha_{i} \psi_{i}^{8,0}$ (arbitrary superposition) and likewise for $\left|1_{L}\right\rangle=\sum_{i} \beta_{i} \psi_{i}^{8,1}$. Using the notation of Sec. IIIC the logical states can be fully identified by the quantum numbers, $\left|p, q ; \lambda ; t, t_{3}, y\right\rangle$, where $p, q$ are the principal quantum numbers which identify the irreducible representation, $\lambda$ is the degeneracy label, and $t, t_{3}, y$ identify the states within the representation by its secondary quantum numbers. The states of the octet which comprise the logical zero state are, in this notation, given by

$$
\begin{aligned}
\psi_{1}^{8,0} & =|1,1 ; 0 ; 1,1,0\rangle, \\
\psi_{2}^{8,0} & =|1,1 ; 0 ; 1 / 2,1 / 2,1\rangle, \\
\psi_{3}^{8,0} & =|1,1 ; 0 ; 1 / 2,-1 / 2,1\rangle, \\
\psi_{4}^{8,0} & =|1,1 ; 0 ; 1,-1,0\rangle, \\
\psi_{5}^{8,0} & =|1,1 ; 0 ; 1 / 2,-1 / 2,-1\rangle, \\
\psi_{6}^{8,0} & =|1,1 ; 0 ; 1 / 2,1 / 2,-1\rangle, \\
\psi_{7}^{8,0} & =|1,1 ; 0 ; 1,0,0\rangle, \\
\psi_{8}^{8,0} & =|1,1 ; 0 ; 0,0,0\rangle .
\end{aligned}
$$


The states which comprise the logical one are given by

$$
\begin{aligned}
& \psi_{1}^{8,1}=|1,1 ; 1 ; 1,1,0\rangle \\
& \psi_{2}^{8,1}=|1,1 ; 1 ; 1 / 2,1 / 2,1\rangle, \\
& \psi_{3}^{8,1}=|1,1 ; 1 ; 1 / 2,-1 / 2,1\rangle, \\
& \psi_{4}^{8,1}=|1,1 ; 1 ; 1,-1,0\rangle, \\
& \psi_{5}^{8,1}=|1,1 ; 1 ; 1 / 2,-1 / 2,-1\rangle, \\
& \psi_{6}^{8,1}=|1,1 ; 1 ; 1 / 2,1 / 2,-1\rangle, \\
& \psi_{7}^{8,1}=|1,1 ; 1 ; 1,0,0\rangle \\
& \psi_{8}^{8,1}=|1,1 ; 1 ; 0,0,0\rangle .
\end{aligned}
$$

The remaining 11 states include a (completely antisymmetric) singlet

$$
\psi_{s}=(|012\rangle-|021\rangle-|102\rangle+|120\rangle-|210\rangle) / \sqrt{6}
$$

and a (completely symmetric) decuplet of states:

$$
\begin{aligned}
& \psi_{1}^{10}=|111\rangle \\
& \psi_{2}^{10}=(|011\rangle+|101\rangle+|110\rangle) / \sqrt{3} \\
& \psi_{3}^{10}=(|001\rangle+|010\rangle+|100\rangle) / \sqrt{3} \\
& \psi_{4}^{10}=|000\rangle \\
& \psi_{5}^{10}=(|112\rangle+|121\rangle+|211\rangle) / \sqrt{3} \\
& \psi_{6}^{10}=(|012\rangle+|021\rangle+|102\rangle+|120\rangle+|210\rangle) / \sqrt{6} \\
& \psi_{7}^{10}=(|002\rangle+|020\rangle+|200\rangle) / \sqrt{3} \\
& \psi_{8}^{10}=(|122\rangle+|212\rangle+|221\rangle) / \sqrt{3} \\
& \psi_{9}^{10}=(|022\rangle+|202\rangle+|220\rangle) / \sqrt{3} \\
& \psi_{10}^{10}=|222\rangle
\end{aligned}
$$

A basis for the collective errors for qutrit states is given by sums of the operators $\left\{\lambda_{i}\right\}$ of Appendix

$$
S^{\alpha}=\sum_{i} \lambda_{i}^{\alpha},
$$

where $\alpha=1,2, \ldots, 8$ and $i$ denotes the physical qutrit 1,2 or 3. A generic collective error has the form

$$
S=\sum_{\alpha} a_{\alpha} S^{\alpha}
$$

where the $a_{\alpha}$ are arbitrary constants. As in the three qubit DFS/NS, the states within blocks $\left|0_{L}\right\rangle$ and $\left|1_{L}\right\rangle$ get mixed with each other in exactly the same way during collective operations, but states in one block are not mixed with states in another. Logical operations will mix these blocks with each other. In the DFS/NS basis, the operators $S_{\mathrm{dfs}}^{\alpha}=V_{\mathrm{dfs}} S^{\alpha} V_{\mathrm{dfs}}^{-1}$ are block diagonal in accordance with Eq. (10). Let us order the states in a column vector: $\Psi=\operatorname{column}\left\{\psi_{1}^{8,0}, \psi_{2}^{8,0}, \psi_{3}^{8,0}, \psi_{4}^{8,0}, \psi_{5}^{8,0}, \psi_{6}^{8,0}\right.$, $\psi_{7}^{8,0}, \psi_{8}^{8,0}, \psi_{1}^{8,1}, \psi_{2}^{8,1}, \psi_{3}^{8,1}, \psi_{4}^{8,1}, \psi_{5}^{8,1}, \psi_{6}^{8,1}, \psi_{7}^{8,1}, \psi_{8}^{8,1}$, $\left.\psi_{s}, \psi_{1}^{10}, \psi_{2}^{10}, \psi_{3}^{10}, \psi_{4}^{10}, \psi_{5}^{10}, \psi_{6}^{10}, \psi_{7}^{10}, \psi_{8}^{10}, \psi_{9}^{10}, \psi_{10}^{10}\right\}$. From these states one may readily deduce the transformation $V_{\mathrm{dfs}}$ which takes the qutrit computational basis states to the DFS/NS basis. It is then clear that $V_{\mathrm{dfs}}$ is a $27 \times 27$ matrix of $S U(3)$ Wigner-Clebsch-Gordan coefficients. Since, the collective errors in this basis are block diagonal [viz. Eq. (10)], these blocks will be labeled according to the set of states on which they act nontrivially. Let $S_{0}$ be the first such block (which acts nontrivially on the states which form the logical zero), $S_{1}$ be the second such block (which acts nontrivially on the states which form the logical one), $S_{s}$ be the third such block (which acts on the singlet state), and $S_{10}$ which acts on the states in the decuplet. The form of the matrix $S_{\mathrm{dfs}}=V_{\mathrm{dfs}} S V_{\mathrm{dfs}}^{-1}$ is given by

$$
S_{\mathrm{dfs}}=\left(\begin{array}{cccc}
S_{0} & 0 & 0 & 0 \\
0 & S_{1} & 0 & 0 \\
0 & 0 & S_{s} & 0 \\
0 & 0 & 0 & S_{10}
\end{array}\right)
$$

where $S_{0}$ and $S_{1}$ are both $8 \times 8$ matrices and are given by

$$
\left(\begin{array}{cccccccc}
2 a_{3} & a_{6}+i a_{7} & 0 & 0 & 0 & a_{4}-i a_{5} & \sqrt{2}\left(a_{1}-i a_{2}\right) & 0 \\
a_{6}-i a_{7} & a_{3}+\sqrt{3} a_{8} & a_{1}-i a_{2} & 0 & 0 & 0 & \frac{-a_{4}+i a_{5}}{\sqrt{2}} & \frac{-3\left(a_{4}-i a_{5}\right)}{\sqrt{6}} \\
0 & a_{1}+i a_{2} & -a_{3}+\sqrt{3} a_{8} & -a_{4}+i a_{5} & 0 & 0 & \frac{a_{6}-i a_{7}}{\sqrt{2}} & \frac{-3\left(a_{6}-i a_{7}\right)}{\sqrt{6}} \\
0 & 0 & -a_{4}-i a_{5} & -2 a_{3} & a_{6}-i a_{7} & 0 & \sqrt{2}\left(a_{1}+i a_{2}\right) & 0 \\
0 & 0 & 0 & a_{6}+i a_{7} & -a_{3}-\sqrt{3} a_{8} & a_{1}+i a_{2} & \frac{a_{4}+i a_{5}}{\sqrt{2}} & \frac{3\left(a_{4}+i a_{5}\right)}{\sqrt{6}} \\
a_{4}+i a_{5} & 0 & 0 & 0 & a_{1}-i a_{2} & a_{3}-\sqrt{3} a_{8} & \frac{a_{6}+i a_{7}}{\sqrt{2}} & \frac{-3\left(a_{6}+i a_{7}\right)}{\sqrt{6}} \\
\sqrt{2}\left(a_{1}+i a_{2}\right) & \frac{-a_{4}-i a_{5}}{\sqrt{2}} & \frac{a_{6}+i a_{7}}{\sqrt{2}} & \sqrt{2}\left(a_{1}-i a_{2}\right) & \frac{a_{4}-i a_{5}}{\sqrt{2}} & \frac{a_{6}-i a_{7}}{\sqrt{2}} & 0 & 0 \\
0 & \frac{-3\left(a_{4}+i a_{5}\right)}{\sqrt{6}} & \frac{-3\left(a_{6}+i a_{7}\right)}{\sqrt{6}} & 0 & \frac{3\left(a_{4}-i a_{5}\right)}{\sqrt{6}} & \frac{-3\left(a_{6}-i a_{7}\right)}{\sqrt{6}} & 0 & 0
\end{array}\right)
$$

The matrix $S_{s}$ is a $1 \times 1$ zero "matrix" and the $10 \times 10$

matrix $S_{10}$ will not be displayed since it is not relevant 
for the DFS/NS.

In summary, if the physical circumstances are such that the errors/operations on a set of three qutrits in the 3 representation are identical on each qutrit, then errors/operators will have the form, of Eq.(36). There is then a two-state subsystem formed by two collections of states $\psi_{i}^{8,0}$ and $\psi_{j}^{8,1}$ which may represent a decoherencefree, or noiseless, subsystem.

\section{DISCUSSION AND CONCLUSIONS}

We note that this research was prompted, in part, by the following question: How does a quantum state or operator transform? This is a fundamental, physically motivated question. The transformation properties determine the good quantum numbers of a state. For three-state, and higher-dimensional systems, the states could transform in one of two ways under special unitary transformations and the representations are inequivalent. There are several physical consequences of the difference in transformation properties.

For the theory of decoherence-free, or noiseless, subsystems it is important to determine the transformation properties which distinguish different physical states. Without this knowledge, it is not possible to reliably form a DFS/NS. Using Weyl's unitary trick, this is clearly seen through the use of Young's tableau for analyzing the irreps of the $S U(d)$ groups.

Similarly, simulating quantum systems with other quantum systems requires strict adherence to the appropriate transformation rules during the applications of quantum controls. A very important example of this is provided by low-energy nuclear interactions and the quark model. Quark-quark interactions at low energies involve both weak and strong forces. Both the theories of strong and weak forces involve $S U(d)$ symmetry groups. QCD is a non-Abelian gauge theory, with gauge group $S U(3)$. In this theory, quarks transform according to the 3 rep of the group $S U(3)$. Weak interaction physics, or "flavor" physics, potentially involves six flavors of quarks which have an approximate $S U(6)$ symmetry. At lower energies, only the quarks with mass less than the experimental interaction energies are used in calculations. The three lightest quarks are quite close in mass and have an approximate $S U(3)$ symmetry to an even better approximation than the $S U(6)$ theory. (Reference [51] contains detailed discussions of these topics.) Whereas quarks transform according to the $\mathbf{3}$ rep, antiquarks transform according to the $\overline{\mathbf{3}}$ rep. Baryons, such as the proton and neutron, are color-neutral bound states of three quarks. Mesons behave as color-neutral states of quarks and antiquarks. Thus the transformation properties of the particles involved in low energy nuclear interactions are critically important in simulations. Since there exist states of particles which behave in such a way,the differences in transformation properties of quantum systems must be taken into account during the simulation of low-energy nuclear physics.

We may therefore conclude that the existence of inequivalent fundamental irreps for $S U(d)$ can be vital for quantum information processing, whether the systems being used to process quantum information contain $d$ distinct orthogonal states, or a system being simulated contains $d$ such states. Clearly there is a great deal of work still to be done in this area. Whether or not a system transforms according to a barred or unbarred representation is determined by the physical system. Not all systems will naturally obey a super-selection rule of this sort.

In the near future, we anticipate exploring quantum computing in DFS/NSs constructed from these higherdimensional state spaces. We also expect to more fully explore the experimental circumstances which give rise to DFS/NSs and how one would control the system to keep it in a DFS/NS.

\section{Acknowledgments}

The author thanks ORDA of Southern Illinois University for partial support of this work under internal grant 4-14095 and Centro de Ciências Matemáticas at the University of Madeira. This work was supported in part by POPRAM III and CITMA, Portugal, and was undertaken during the XXIX Madeira Math encounter. The author also thanks V. Akulin, J. Clark, A. Mandilara, and especially N. Harshman for stimulating and helpful discussions.

\section{APPENDIX A: GROUP THEORY AND YOUNG TABLEAU}

\section{Group theory primer}

In this appendix various definitions are collected which may or may not be familiar to the reader. Since only matrix representations are considered here, one should place the word "matrix" in front of "representation" throughout the article to be precise.

If there exists a homomorphic mapping of a group $\mathcal{G}$ onto a group of nonsingular $d \times d$ matrices $\Gamma(T)$, for all $T \in \mathcal{G}$ then the set of matrices $\Gamma(T)$ forms a $d$ dimensional (matrix) representation $\Gamma$ of the group $\mathcal{G}$. If the homomorphism is also an isomorphism (one to one and onto) then the representation is said to be faithful. Here representations of $S U(d)$ will be considered. The lowest-dimensional set of matrices which faithfully represents it contains $d \times d$ matrices. A faithful representation of $S U(d)$ by $d \times d$ matrices is called a fundamental representation and is necessarily irreducible. (We will take "irreducible representation" to mean that the representation cannot be written in terms of matrices of smaller dimension 52].) Two representations, $\Gamma$ and $\Gamma^{\prime}$, of a group $\mathcal{G}$ are said to be equivalent, if there exists a similarity 
transformation $S$ such that

$$
\Gamma^{\prime}=S \Gamma S^{-1}
$$

for all $\Gamma \in \mathcal{G}$. If there is no such transformation, then the two representations are said to be inequivalent.

Theorem There are two inequivalent irreducible fundamental representations of $S U(d)$ for $d \geq 3$.

\section{Young's tableau}

Here, a brief summary of some properties of Young tableau are given.

Young's tableau enable the determination of the irreducible components of a tensor product. These methods are used in the text to find the direct sum of irreps arising from a tensor product of irreps. For a more detailed explanation and derivation of the rules, consult a text on group theory-for example [34, 35]. Since only $S U(d)$ representations (and corresponding equivalent algebraic representations) are used in this article, the rules are discussed in terms of $S U(d)$ irreps.

For representations of $S U(N)$, a single box $\square$ corresponds to a fundamental irreducible representation. The dimension of the representation is determined by filling the box with numbers $1,2, \ldots, N$.

A set of two boxes corresponding to two antisymmetric indices under their interchange is provided by vertical boxes: $\square$. Similarly, two horizontal boxes, $\square$, represent indices which are symmetric under interchange. In general, the indices must be antisymmetric under interchange of numbers in columns of the tableau and symmetric in the rows.

To determine the number of states in an irrep, the boxes are filled with numbers. For example there are $N$ states in the fundamental irrep of $S U(N)$. Thus different integers $1,2, \ldots, N$ can be put into the boxes, each set of numbers in the boxes representing a different state within the irrep. In order to prevent overcounting, the boxes are filled such that the numbers are nondecreasing from left to right. Filling boxes which are above one another requires the numbers to be distinct for a nonzero tableau due to the antisymmetry. In addition, overcounting will be prevented if the numbers are filled in a strictly increasing order from top to bottom.

A familiar example is provided in Sec. IIIC where the product of two two-state systems is shown to produce a singlet and a triplet using Young's Tableau.

\section{Singlet states}

Here, a set of raising and lowering operators are given for $S U(3)$ in terms of the Gell-Mann matrices. It is also shown that the state $\left|\phi^{\prime}\right\rangle=(1 / \sqrt{3}) \sum_{i}|i \bar{i}\rangle$ is a singlet state.
There are several ways to show that $\left|\phi^{\prime}\right\rangle$ is a singlet state. To do it directly from relations (26), one can use the relations for the raising and lower operators on the states. In terms of the Gell-Mann matrices,

$$
\begin{aligned}
T_{ \pm} & =\frac{1}{2}\left(\lambda_{1} \pm i \lambda_{2}\right), \quad T_{3}=\frac{1}{2} \lambda_{3}, \\
V_{ \pm} & =\frac{1}{2}\left(\lambda_{4} \pm i \lambda_{5}\right), \quad U_{ \pm}=\frac{1}{2}\left(\lambda_{6} \pm i \lambda_{7}\right), \\
Y & =\frac{1}{\sqrt{3}} \lambda_{8} .
\end{aligned}
$$

One may also define $V_{3}=(1 / 2)\left(\sqrt{3} \lambda_{8}+T_{3}\right)$ and $U_{3}=$ $(1 / 2)\left(\sqrt{3} \lambda_{8}-T_{3}\right)$. This proves convenient since one may use the known raising and lowering operation relations of $S U(2) ; \lambda_{4}, \lambda_{5}, V_{3}$ and $\lambda_{6}, \lambda_{7}, U_{3}$ each form an $S U(2)$ algebra. For a given $p, q$, the states of an irrep may be labeled using $t, t_{3}$, and $y$. The states are eigenstates of $T_{3}$ and $Y$ by construction,

$$
T_{3}\left|t, t_{3}, y\right\rangle=t_{3}\left|t, t_{3}, y\right\rangle, \quad Y\left|t, t_{3}, y\right\rangle=y\left|t, t_{3}, y\right\rangle .
$$

At this point one would proceed essentially as is done for finding the raising and lowering operator relations for $S U(2)$. However, this is somewhat tedious and there is an easier way.

A singlet representation transforms trivially under actions of the group, as a scalar. Therefore, if one notes that the qutrit states transform according to the representation $U$, for $i=0,1,2$,

$$
|i\rangle \rightarrow \sum_{j} U_{i j}|j\rangle,|i\rangle \in \mathbf{3},
$$

and the barred states transform according to the representation $U^{*}$, the complex conjugate of $U$,

$$
|\bar{i}\rangle \rightarrow \sum_{k} U_{i k}^{*}|\bar{k}\rangle,|\bar{i}\rangle \in \overline{\mathbf{3}}
$$

then, under the transformation of each,

$$
\begin{aligned}
\sum_{i}|i\rangle|\bar{i}\rangle \rightarrow & \sum_{i j k} U_{i j}|j\rangle U_{i k}^{*}|\bar{k}\rangle \\
& =\sum_{i j k} U_{i j}\left(U^{\dagger}\right)_{k i}|j\rangle|\bar{k}\rangle \\
& =\sum_{j k} \delta_{j k}|j\rangle|\bar{k}\rangle \\
& =\sum_{k}|k\rangle|\bar{k}\rangle .
\end{aligned}
$$

Therefore the state $\sum_{k}|k\rangle|\bar{k}\rangle$ is an $S U(3)$ scalar. It is not difficult to convince oneself that this implies that the raising and lowering operators acting on this state give zero. (Yet another proof uses the explicit parameterization and differential operators found in [53].) The eigenvalues of $T_{3}$ and $Y$ are clearly zero since these act as derivations,

$$
\mathcal{O} \sum_{i}|i \bar{i}\rangle=\sum_{i}(\mathcal{O}|i\rangle)|\bar{i}\rangle+|i\rangle(\mathcal{O}|\bar{i}\rangle)=0,
$$


and for any operator in the Lie algebra and the eigenvalues of barred states are opposite those of the unbarred states.

\section{APPENDIX B: THE ALGEBRA OF $S U(3)$}

In this appendix the Gell-Mann matrices are listed. This is one basis for the algebra which is commonly used for the subgroup chain that is discussed throughout the article. However, it should be emphasized that this is not the only basis one could choose. The Casimir operators are also given for $S U(3)$ since they are elements of the CSCO discussed in the text.

The Gell-Mann matrices are given by

$$
\begin{aligned}
& \lambda_{1}=\left(\begin{array}{lll}
0 & 1 & 0 \\
1 & 0 & 0 \\
0 & 0 & 0
\end{array}\right), \lambda_{2}=\left(\begin{array}{ccc}
0 & -i & 0 \\
i & 0 & 0 \\
0 & 0 & 0
\end{array}\right), \\
& \lambda_{3}=\left(\begin{array}{rrr}
1 & 0 & 0 \\
0 & -1 & 0 \\
0 & 0 & 0
\end{array}\right), \lambda_{4}=\left(\begin{array}{lll}
0 & 0 & 1 \\
0 & 0 & 0 \\
1 & 0 & 0
\end{array}\right), \\
& \lambda_{5}=\left(\begin{array}{rrr}
0 & 0 & -i \\
0 & 0 & 0 \\
i & 0 & 0
\end{array}\right), \lambda_{6}=\left(\begin{array}{lll}
0 & 0 & 0 \\
0 & 0 & 1 \\
0 & 1 & 0
\end{array}\right),
\end{aligned}
$$

$$
\lambda_{7}=\left(\begin{array}{ccc}
0 & 0 & 0 \\
0 & 0 & -i \\
0 & i & 0
\end{array}\right), \lambda_{8}=\frac{1}{\sqrt{3}}\left(\begin{array}{ccc}
1 & 0 & 0 \\
0 & 1 & 0 \\
0 & 0 & -2
\end{array}\right)
$$

The Casimir operators for $S U(3)$ are proportional to the operator which is quadratic in the algebraic elements,

$$
C_{2}=\sum_{i=1}^{8} \lambda_{i}^{2}
$$

and the operator which is cubic in the algebraic elements,

$$
C_{3}=\sum_{i=1}^{8} d_{i j k} \lambda_{i} \lambda_{j} \lambda_{k}
$$

where

$$
d_{i j k}=\frac{1}{4} \operatorname{Tr}\left(\left[\lambda_{i} \lambda_{j}+\lambda_{j} \lambda_{i}\right] \lambda_{k}\right) .
$$

These can be written in terms of functions of $p$ and $q$ using the operators $T_{3}, Y$ and the raising and lower operators 39].
[1] C.M. Caves and G.J. Milburn, Opt. Commun. 179, 439 (2000).

[2] P. Rungta, W. J. Munro, K. Nemoto, P. Deuar, G. J. Milburn, and C. M. Caves, in Directions in Quantum Optics: A Collection of Papers Dedicated to the Memory of Dan Walls, edited by H. J. Carmichael, R. J. Glauber, and M. O. Scully (Springer-Verlag, Berlin, 2000), p. 149.

[3] J.-L. Chen, D. Kaszlikowski, L. C. Kwek, C. H. Oh and M. Zukowski, Phys. Rev. A 64, 052109 (2001).

[4] K.A. Dennison and W.K. Wootters, Phys. Rev. A 65, 010301(R) (2001).

[5] A. Kitaev, D. Mayers and J. Preskill, Phys. Rev. A 69, 052326 (2004).

[6] G.-C. Wick, A.S. Wightman and E.P. Wigner, Phys. Rev. 88, 101 (1952).

[7] Y. Aharanov and L. Susskind, Phys. Rev. 155, 1428 (1967).

[8] G.-C. Wick, A.S. Wightman and E.P. Wigner, Phys. Rev. D 1, 3267 (1970).

[9] F. Verstraete and J.I. Cirac, Phys. Rev. Lett. 91, 010404 (2003).

[10] S.D. Bartlett and H.M. Wiseman, Phys. Rev. Lett. 91, 097903 (2003).

[11] D. Mayers (2002), quant-ph/0212159.

[12] S.D. Bartlett, A.C. Doherty, R.W. Spekkens, H.M. Wiseman (2004), quant-ph/0412158.

[13] P. Zanardi and M. Rasetti, Phys. Rev. Lett. 79, 3306 (1997).
[14] L.-M Duan and G.-C. Guo, Phys. Rev. A 57, 737 (1998).

[15] D.A. Lidar, I.L. Chuang and K.B. Whaley, Phys. Rev. Lett. 81, 2594 (1998).

[16] E. Knill, R. Laflamme and L. Viola, Phys. Rev. Lett. 84, 2525 (2000).

[17] J. Kempe, D. Bacon, D.A. Lidar, and K.B. Whaley, Phys. Rev. A 63, 042307 (2001).

[18] D.A. Lidar, D. Bacon, J. Kempe, and K.B. Whaley, Phys. Rev. A 63, 022306 (2001).

[19] D.A. Lidar and K.B. Whaley, in Irreversible Quantum Dynamics (Springer-Verlag, Berlin, 2003).

[20] M. S. Byrd, L.-A. Wu, D. A. Lidar, J. Mod. Optics 51, 2449 (2004).

[21] D. Bacon, J. Kempe, D. A. Lidar, K. B. Whaley, and D. P. DiVincenzo, in Proceedings of the 1st International Conference on Experimental Implementations of Quantum Computation, edited by R. Clark (Rinton, Princeton, NJ, 2001), p. 257.

[22] D.P. DiVincenzo, D. Bacon, J. Kempe, G. Burkard, and K. B. Whaley, Nature 408, 339 (2000).

[23] J. Levy, Phys. Rev. Lett. 89, 147902 (2002).

[24] D.A. Lidar and L.-A. Wu, Phys. Rev. Lett. 88, 017905 (2002).

[25] M. S. Byrd and D. A. Lidar, Phys. Rev. Lett. 89, 047901 (2002).

[26] L.-A. Wu, M.S. Byrd, D.A. Lidar, Phys. Rev. Lett. 89, 127901 (2002).

[27] M.S. Byrd, D.A. Lidar, L.-A. Wu and P. Zanardi, Phys. 
Rev. A 71, 052301(2005).

[28] L. Tian and S. Lloyd, Phys. Rev. A 62, 050301(R) (2000).

[29] S.S. Bullock, D.P. O'Leary and G.K. Brennen, Phys. Rev. Lett. 94, 230502 (2005).

[30] G.K. Brennen, D.P. O'Leary and S.S. Bullock, Phys. Rev. A 71, 052318 (2005).

[31] H. Bombin, M.A. Martin-Delgado Phys. Rev. A 72, 032313 (2005).

[32] J.F. Cornwell, Group Theory in Physics, vol. I and II of Techniques of Physics: Vol. 7 (Academic Press, London, 1984).

[33] J.-S. Huang, Lectures on Representation Theory (World Scientific, River Edge, NJ, 1999).

[34] L.C. Biedenharn and J.D. Louck, Angular Momentum in Quantum Physics: volume 8 of Encyclopedia of Mathematics and Its Applications (Addison-Wesley, 1981).

[35] J.F. Cornwell, Group Theory in Physics, vol. II of Techniques of Physics: 7 (Academic Press, London, 1984).

[36] A. Bohm, Quantum Mechanics: Foundations and Applications, 3rd Ed., Chapter 5 (Springer-Verlag, New York, New York, 1993).

[37] M. Gell-Mann and Y. Ne'eman, The Eightfold Way (Benjamin, New York, 1964).

[38] J.P. Elliott, Proc. Roy. Soc. A 245, 128 (1958).

[39] W. Greiner, B. Müller, Quantum mechanics: Symme- tries, 2nd Ed. (Springer-Verlag, New York, 1994).

[40] A.J. Scott, Phys. Rev. A 69, 052330 (2004).

[41] This is a famous product in particle physics which prompted the name "Eightfold way" 37].

[42] P. Zanardi, Phys. Lett. A 258, 77 (1999).

[43] L. Viola, E. Knill, and S. Lloyd, Phys. Rev. Lett. 85, 3520 (2000).

[44] L.-A. Wu and D. A. Lidar, Phys. Rev. Lett. 88, 207902 (2002).

[45] L. Viola, Phys. Rev. A 66, 012307 (2002).

[46] M. S. Byrd and D.A. Lidar, Qu. Inf. Proc. 1, 19 (2001).

[47] M.S. Byrd and D.A. Lidar, Phys. Rev. A 67, 012324 (2003).

[48] M.S. Byrd and D.A. Lidar, J. Mod. Optics 50, 1285 (2002).

[49] D.A. Lidar and L.-A Wu, Phys. Rev. A 67, 032313 (2003).

[50] P. Zanardi, Phys. Rev. A 60, R729 (1999).

[51] S. Weinberg, The Quantum Theory of Fields, Vol. II (Cambridge, New York, 1995).

[52] The formal definition has been omitted here. See for example, [32], pages 76-83.

[53] M. S. Byrd and E.C.G. Sudarshan, J. Phys. A 31, 9255 (1998). 